\title{
Asset Prices, Liquidity, and Monetary Policy in the Search Theory of Money
}

Ricardo Lagos

\begin{abstract}
The author presents a search-based model in which money coexists with equity shares on a risky aggregate endowment. Agents can use equity as a means of payment, so shocks to equity prices translate into aggregate liquidity shocks that disrupt the mechanism of exchange. The author characterizes a family of optimal monetary policies and finds that the resulting equity prices are independent of monetary considerations. The author also studies monetary policies that target a constant, but nonzero, nominal interest rate and finds that to the extent that a financial asset is valued as a means to facilitate transactions, the asset's real rate of return will include a liquidity return that depends on monetary considerations. Through this liquidity channel, persistent deviations from an optimal monetary policy can cause the real prices of assets that can be used to relax trading constraints to exhibit persistent deviations from their fundamental values. (JEL E31, E52, G12)
\end{abstract}

Federal Reserve Bank of St. Louis Review, July/August 2010, 92(4), 303-09.

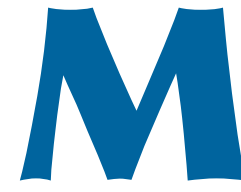

any financial assets are held not only for the intrinsic value of the stream of consumption that they yield, but also for their usefulness in facilitating exchange. Consider a buyer who cannot commit to or be forced to honor debts and who wishes to make a purchase from a seller. This buyer would find any asset that is valuable to the seller (e.g., an equity share, a bond, money) helpful to carry out the transaction. For example, the buyer could settle the transaction on the spot by using the asset directly as a means of payment. In some modern transactions, often the buyer uses a financial asset to enter a repurchase agreement with the seller or as collateral to borrow the funds needed to pay the seller. Once stripped from the subsidiary contractual complexities, the essence of these transactions is that the asset helps the untrustworthy buyer to obtain what he wants from the seller. In this sense, many financial assets are routinely used in the exchange process and play a role akin to a medium of exchange-that is, they provide liquidity-the term that monetary theorists use to refer to the usefulness of an asset in facilitating transactions.

Financial assets are subject to price fluctuations resulting from aggregate shocks; therefore, to the extent that these assets serve as a source of liquidity, shocks to their prices translate into aggregate liquidity shocks that disrupt the mechanism of exchange and the ensuing allocations. Recent developments in financial markets have renewed economists' interest in the idea that fluctuations in asset prices can disrupt the exchange process in some key markets and, through this channel, propagate to the macroeconomy.

Much of the policy advice offered to central banks is framed in terms of simple interest rate feedback rules loosely motivated by a particular class of models in which the preeminent friction is a specific type of reduced-form nominal rigidity. Such policy recommendations are based on

Ricardo Lagos is an associate professor of economics at New York University. Financial support from the C.V. Starr Center for Applied Economics at New York University is gratefully acknowledged.

(C) 2010, The Federal Reserve Bank of St. Louis. The views expressed in this article are those of the author(s) and do not necessarily reflect the views of the Federal Reserve System, the Board of Governors, or the regional Federal Reserve Banks. Articles may be reprinted, reproduced, published, distributed, displayed, and transmitted in their entirety if copyright notice, author name(s), and full citation are included. Abstracts, synopses, and other derivative works may be made only with prior written permission of the Federal Reserve Bank of St. Louis. 
the premise that the primary goal of monetary policy is to mitigate the effects of these rigidities. With no room or role for a notion of liquidity (and typically even no meaningful role for money), this conventional view that dominates policy circles has failed to offer relevant policy guidance in the midst of the recent financial crisis. I interpret this failure as an indication that the consensus stance toward monetary policy, with its theoretical focus on sticky price frictions and its implementation emphasis on ad hoc feedback interest rate rules, is too narrow in that it neglects the fundamental frictions that give rise to a demand for liquidity.

In this article, I present a dynamic equilibrium, microfounded monetary asset-pricing framework with multiple assets and aggregate uncertainty regarding liquidity needs, and discuss the main normative and positive policy implications of the theory. The broad view that emerges from explicitly modeling the role of money and other liquid assets in the exchange process is that of a monetary authority that seeks to provide the private sector with the liquidity needed to conduct market transactions. More precisely, I state and explain three propositions that answer the following questions: How should monetary policy be conducted to mitigate the adverse effects of shocks to the valuations of the financial assets that provide liquidity to the private sector? What are the implications for asset prices of deviating from the optimal monetary policy? Are such deviations capable of inflating real asset prices above their fundamental values for extended periods of time?

\section{MODEL}

In this section I outline a bare-bones model that encompasses the key economic mechanisms. ${ }^{1}$ The model combines elements of the asset-pricing model of Lucas (1978) with elements of the model of monetary exchange of Lagos and Wright (2005). Time is discrete and the horizon infinite. There is a $[0,1]$ continuum of infinitely lived agents. Each time period is divided into two subperiods

1 The analysis that follows is based on Lagos $(2006,2009,2010)$. during which different activities take place. There are three nonstorable and perfectly divisible consumption goods at each date: fruit, general goods, and special goods. Fruit and general goods are homogeneous goods, whereas special goods come in many varieties. The only durable commodity in the economy is a set of "Lucas trees." The number of trees is fixed and equal to the number of agents. Trees yield (the same amount of) a random quantity $x_{t}$ of fruit in the second subperiod of every period $t$. The realization of $x_{t}$ becomes known to all at the beginning of period $t$ (when agents enter the first subperiod). Production of fruit is entirely exogenous: No resources are used and it is not possible to affect the output at any time. The motion of $x_{t}$ is assumed to follow a Markov process, defined by its transition function

$$
F\left(x^{\prime}, x\right)=\operatorname{Pr}\left(x_{t+1} \leq x^{\prime} \mid x_{t}=x\right) .
$$

For each fixed $x, F(\cdot, x)$ is a distribution function with support $\Xi \subseteq(0, \infty)$.

In each subperiod, every agent is endowed with $\bar{n}$ units of time that can be used as labor services. In the second subperiod, each agent has access to a linear production technology that transforms labor services into general goods. In the first subperiod, each agent has access to a linear production technology that transforms his own labor input into a particular variety of the special good that the agent does not consume. This specialization is modeled as follows: Given two agents $i$ and $j$ drawn at random, there are three possible events. The probability that $i$ consumes the variety of special good that $j$ produces but not vice versa (a single coincidence) is denoted $\alpha$. Symmetrically, the probability that $j$ consumes the special good that $i$ produces but not vice versa is also $\alpha$. In a single-coincidence meeting, the agent who wishes to consume is the buyer, and the agent who produces is the seller. The probability that neither wants what the other can produce is $1-2 \alpha$, with $\alpha \leq 1 / 2$. Fruit and general goods are homogeneous and hence consumed (and in the case of general goods, also produced) by all agents.

In the first subperiod, agents participate in a decentralized market where trade is bilateral (each meeting is a random draw from the set of pairwise meetings), and the terms of trade are 
determined by bargaining (a take-it-or-leave-it offer by the buyer, for simplicity). The specialization of agents over consumption and production of the special good, combined with bilateral trade, creates a double-coincidence-of-wants problem in the first subperiod. In the second subperiod, agents trade in a centralized market. Agents cannot make binding commitments, and trading histories are private in a way that precludes any borrowing and lending between people, so all trade-both in the centralized and decentralized markets-must be quid pro quo.

Each tree has outstanding one durable and perfectly divisible equity share that represents the bearer's ownership and confers to the owner the right to collect the fruit dividends. A second financial asset, money, is intrinsically useless (it is not an argument of any utility or production function), and unlike equity, ownership of money does not constitute a right to collect any resources. Money is issued by a "government" that at $t=0$ commits to a monetary policy represented by a sequence of positive real-valued functions, $\left\{\mu_{t}\right\}_{t=0}^{\infty}$. Given an initial stock of money, $M_{0}>0$, a monetary policy induces a money supply process, $\left\{M_{t}\right\}_{t=0}^{\infty}$, by means of $M_{t+1}=\mu_{t}\left(x^{t}\right) M_{t}$, where $x^{t}$ denotes a history of realizations of fruit dividends through period $t$-that is, $x^{t}=\left(x_{t}, x_{t-1}, \ldots, x_{0}\right)$. The government injects or withdraws money through lumpsum transfers or taxes in the second subperiod of every period; thus, along every sample path,

$M_{t+1}=M_{t}+T_{t}$, where $T_{t}$ is the lump-sum transfer (or tax, if negative). All assets are perfectly recognizable, cannot be forged, and can be traded among agents in both the centralized and decentralized markets. At $t=0$, each agent is endowed with $a_{0}^{s}$ equity shares and $a_{0}^{m}$ units of fiat money.

Let the utility function for special goods, $u: \mathbb{R}^{+} \rightarrow \mathbb{R}^{+}$, and the utility function for fruit, $U: \mathbb{R}^{+} \rightarrow \mathbb{R}^{+}$, be continuously differentiable, bounded by $B$ on $\Xi$, increasing, and strictly concave, with $u(0)=U(0)$. Let $-n$ be the utility from working $n$ hours in the first subperiod. Also, suppose there exists $q^{*} \in(0, \infty)$ defined by $u^{\prime}\left(q^{*}\right)=1$, with $q^{*} \leq \bar{n}$. Let both the utility for general goods and the disutility from working in the second subperiod be linear. The agent prefers a sequence $\left\{q_{t}, n_{t}, c_{t}, y_{t}, h_{\tilde{t}}\right\}_{t=0}^{\infty}$ over another sequence $\left\{\tilde{q}_{t}, \tilde{n}_{t}, \tilde{c}_{t}, \tilde{y}_{t}, \tilde{h}_{t}\right\}_{t=0}^{\infty}$ if

$$
\begin{aligned}
& \liminf _{T \rightarrow \infty} E_{0} \sum_{t=0}^{T}\left\{\beta^{t}\left[u\left(q_{t}\right)-n_{t}+U\left(c_{t}\right)+y_{t}-h_{t}\right]\right. \\
&\left.-\beta^{t}\left[u\left(\tilde{q}_{t}\right)-\tilde{n}_{t}+U\left(\tilde{c}_{t}\right)+\tilde{y}_{t}-\tilde{h}_{t}\right]\right\} \geq 0,
\end{aligned}
$$

where $\beta \in(0,1), q_{t}$ and $n_{t}$ are the quantities of special goods consumed and produced in the decentralized market, $c_{t}$ denotes consumption of fruit, $y_{t}$ consumption of general goods, $h_{t}$ the hours worked in the second subperiod, and $E_{t}$ is an expectations operator conditional on the information available to the agent at time $t$, defined with respect to the matching probabilities and the probability measure induced by $F$.

Next, consider the individual optimization problems. Let $\boldsymbol{a}_{t}=\left(a_{t}^{s}, a_{t}^{m}\right)$ denote the portfolio of an agent who holds $a_{t}^{s}$ shares and $a_{t}^{m}$ units of money. Let $W_{t}\left(\boldsymbol{a}_{t}\right)$ and $V_{t}\left(\boldsymbol{a}_{t}\right)$ be the maximum attainable expected discounted utility of an agent who enters the centralized and decentralized market, respectively, at time $t$ with portfolio $\boldsymbol{a}_{t}$. Then,

$$
\begin{aligned}
& W_{t}\left(\boldsymbol{a}_{t}\right)= \\
& \max _{c_{t}, y_{t}, h_{t}, \boldsymbol{a}_{t+1}}\left\{U\left(c_{t}\right)+y_{t}-h_{t}+\beta E_{t} V_{t+1}\left(\boldsymbol{a}_{t+1}\right)\right\}
\end{aligned}
$$

$$
\begin{aligned}
& \text { s.t. } c_{t}+w_{t} y_{t}+\phi_{t}^{s} a_{t+1}^{s}+\phi_{t}^{m} a_{t+1}^{m}= \\
& \left(\varphi_{t}^{s}+x_{t}\right) a_{t}^{s}+\phi_{t}^{m}\left(a_{t}^{m}+T_{t}\right)+w_{t} h_{t} \\
& 0 \leq c_{t}, 0 \leq h_{t} \leq \bar{n}, 0 \leq \boldsymbol{a}_{t+1} .
\end{aligned}
$$

The agent chooses consumption of fruit $\left(c_{t}\right)$, consumption of general goods $\left(y_{t}\right)$, labor supply $\left(h_{t}\right)$, and an end-of-period portfolio $\left(\boldsymbol{a}_{t+1}\right)$. Fruit is used as numéraire: $w_{t}$ is the relative price of the general good, $\phi_{t}^{S}$ is the (ex-dividend) price of a share, and $1 / \phi_{t}^{m}$ is the dollar price of fruit.

Let $\left[q_{t}(\boldsymbol{a}, \tilde{\boldsymbol{a}}), \boldsymbol{p}_{t}(\boldsymbol{a}, \tilde{\boldsymbol{a}})\right]$ denote the terms at which a buyer who owns portfolio $\boldsymbol{a}$ trades with a seller who owns portfolio $\tilde{\boldsymbol{a}}$, where $q_{t}(\boldsymbol{a}, \tilde{\boldsymbol{a}}) \in \mathbb{R}^{+}$ is the quantity of a special good traded, and $\boldsymbol{p}_{t}(\boldsymbol{a}, \tilde{\boldsymbol{a}})=\left[p_{t}^{S}(\boldsymbol{a}, \tilde{\boldsymbol{a}}), p_{t}^{m}(\boldsymbol{a}, \tilde{\boldsymbol{a}})\right] \in \mathbb{R}^{+} \times \mathbb{R}^{+}$is the transfer of assets from the buyer to the seller (the first argument is the transfer of equity). Consider a meeting in the decentralized market of period $t$ between a buyer with portfolio $\boldsymbol{a}_{t}$ and a seller with portfolio $\tilde{\boldsymbol{a}}_{t}$. The terms of trade, $\left(q_{t}, \boldsymbol{p}_{t}\right)$, are determined by Nash bargaining where the buyer has all the bargaining power: 


$$
\begin{aligned}
& \max _{q_{t}, \boldsymbol{p}_{t} \leq \boldsymbol{a}_{t}}\left[u\left(q_{t}\right)+W_{t}\left(\boldsymbol{a}_{t}-\boldsymbol{p}_{t}\right)-W_{t}\left(\boldsymbol{a}_{t}\right)\right] \\
& \text { s.t. } W_{t}\left(\tilde{\boldsymbol{a}}_{t}+\boldsymbol{p}_{t}\right)-q_{t} \geq W_{t}\left(\tilde{\boldsymbol{a}}_{t}\right) .
\end{aligned}
$$

The constraint $\boldsymbol{p}_{t} \leq \boldsymbol{a}_{t}$ indicates that the buyer in a bilateral meeting cannot spend more assets than he owns. Let $\boldsymbol{\lambda}_{t}=\left(\boldsymbol{\lambda}_{t}^{s}, \boldsymbol{\lambda}_{t}^{m}\right)$, with $\boldsymbol{\lambda}_{t}^{s} \equiv\left(\phi_{t}^{s}+x_{t}\right) / w_{t}$ and $\lambda_{t}^{m} \equiv \phi_{t}^{m} / w_{t}$. The bargaining outcome is as follows: If $\boldsymbol{\lambda}_{t} \boldsymbol{a}_{t} \geq q^{*}$, the buyer buys $q_{t}=q^{*}$ in exchange for a vector $\boldsymbol{p}_{t}$ of assets with real value $\boldsymbol{\lambda}_{t} \boldsymbol{p}_{t}=q^{*} \leq \boldsymbol{\lambda}_{t} \boldsymbol{a}_{t}$. Otherwise, he pays the seller $\boldsymbol{p}_{t}=\boldsymbol{a}_{t}$, in exchange for $q_{t}=\boldsymbol{\lambda}_{t} \boldsymbol{a}_{t}$. Hence, the quantity of the special good exchanged is $\min \left(\boldsymbol{\lambda}_{t} \boldsymbol{a}_{t}, q^{*}\right) \equiv q\left(\boldsymbol{\lambda}_{t} \boldsymbol{a}_{t}\right)$, and the real value of the portfolio used as payment is $\boldsymbol{\lambda}_{t} \boldsymbol{p}_{t}\left(\boldsymbol{a}_{t}, \tilde{\boldsymbol{a}}_{t}\right)=q\left(\boldsymbol{\lambda}_{t} \boldsymbol{a}_{t}\right)$.

Given the bargaining solution, the value of search to an agent who enters the decentralized market of period $t$ with portfolio $\boldsymbol{a}_{t}$ can be written as

$$
V_{t}\left(\boldsymbol{a}_{t}\right)=S\left(\boldsymbol{\lambda}_{t} \boldsymbol{a}_{t}\right)+W_{t}\left(\boldsymbol{a}_{t}\right),
$$

where $S(x) \equiv \alpha\{u[q(x)]-q(x)\}$ is the expected gain from trade in the decentralized market. Substitute the budget constraint (equation (1)) and $V_{t}\left(\boldsymbol{a}_{t}\right)$ into the right-hand side of $W_{t}\left(\boldsymbol{a}_{t}\right)$ to arrive at

$$
\begin{aligned}
& W_{t}\left(\boldsymbol{a}_{t}\right)=\boldsymbol{\lambda}_{t} \boldsymbol{a}_{t}+\tau_{t}+\max _{c_{t} \geq 0}\left[U\left(c_{t}\right)-\frac{c_{t}}{w_{t}}\right] \\
& +\max _{\boldsymbol{a}_{t+1} \geq 0}\left\{-\frac{\boldsymbol{\phi}_{t} \boldsymbol{a}_{t+1}}{w_{t}}+\beta E_{t}\left[S\left(\boldsymbol{\lambda}_{t+1} \boldsymbol{a}_{t+1}\right)+W_{t+1}\left(\boldsymbol{a}_{t+1}\right)\right]\right\},
\end{aligned}
$$

where $\tau_{t}=\lambda_{t}^{m} T_{t}$ and $\lambda_{t}=\left(\lambda_{t}^{s}, \lambda_{t}^{m}\right)$.

Given a process $\left\{M_{t}\right\}_{t=0}^{\infty}$, an equilibrium is a plan $\left\{c_{t}, \boldsymbol{a}_{t+1}\right\}_{t=0}^{\infty}$, pricing functions $\left\{w_{t}, \boldsymbol{\phi}_{t}\right\}_{t=0}^{\infty}$, and bilateral terms of trade $\left\{q_{t}, \boldsymbol{p}_{t}\right\}_{t=0}^{\infty}$ such that (i) given prices and the bargaining protocol, $\left\{C_{t}, \boldsymbol{a}_{t+1}\right\}_{t=0}^{\infty}$ solves the agent's optimization problem; (ii) the terms of trade are determined by Nash bargainingthat is, $q_{t}=\min \left(\boldsymbol{\lambda}_{t} \boldsymbol{a}_{t}, q^{*}\right)$ and $\boldsymbol{\lambda}_{t} \boldsymbol{p}_{t}=q_{t}$; and (iii) the centralized market clears-that is, $c_{t}=x_{t}$ and $a_{t+1}^{s}=1$. The equilibrium is monetary if $\phi_{t}^{m}>0$ for all $t$, and in this case the money-market clearing condition is $a_{t+1}^{m}=M_{t+1}$. The market-clearing conditions imply $\left\{c_{t}, a_{t+1}^{s}, a_{t+1}^{m}\right\}_{t=0}^{\infty}=\left\{x_{t}, 1, M_{t+1}\right\}_{t=0}^{\infty}$, $w_{t}=1 / U^{\prime}\left(w_{t}\right)$, and once $\left\{\boldsymbol{\phi}_{t}\right\}_{t=0}^{\infty}$ has been found, $\left\{q_{t}\right\}_{t=0}^{\infty}=\left\{\boldsymbol{\lambda}_{t} \boldsymbol{p}_{t}\right\}_{t=0}^{\infty}=\left\{\min \left(\Lambda_{t+1}, q^{*}\right)\right\}_{t=0}^{\infty}$, where $\Lambda_{t+1} \equiv \lambda_{t+1}^{s}+\lambda_{t+1}^{m} M_{t+1}$. Therefore, given a money supply process $\left\{M_{t}\right\}_{t=0}^{\infty}$ and letting $L\left(\Lambda_{t+1}\right) \equiv$ $\left[1+S^{\prime}\left(\Lambda_{t+1}\right)\right]$, a monetary equilibrium can be summarized by a sequence $\left\{\boldsymbol{\phi}_{t}\right\}_{t=0}^{\infty}$ that satisfies the following necessary and sufficient conditions for individual optimization:

$$
\begin{aligned}
& U^{\prime}\left(x_{t}\right) \phi_{t}^{s}=\beta E_{t}\left[L\left(\Lambda_{t+1}\right) U^{\prime}\left(x_{t+1}\right)\left(\phi_{t+1}^{s}+x_{t+1}\right)\right] \\
& U^{\prime}\left(x_{t}\right) \phi_{t}^{m}=\beta E_{t}\left[L\left(\Lambda_{t+1}\right) U^{\prime}\left(x_{t+1}\right) \phi_{t+1}^{m}\right] \\
& \lim _{t \rightarrow \infty} E_{0}\left[\beta^{t} U^{\prime}\left(x_{t}\right) \phi_{t}^{s}\right]=0 \\
& \lim _{t \rightarrow \infty} E_{0}\left[\beta^{t} U^{\prime}\left(x_{t+1}\right) \phi_{t+1}^{m} M_{t+1}\right]=0 .
\end{aligned}
$$

\section{NORMATIVE RESULTS: OPTIMAL POLICY AND IMPLEMENTATION}

The Pareto optimal allocation in this environment can be found by solving the problem of a social planner who wishes to maximize average (equally weighted across agents) expected utility. The planner chooses a plan $\left\{c_{t}, q_{t}, n_{t}, y_{t}, h_{t}\right\}_{t=0}^{\infty}$ subject to the feasibility constraints-that is, $0 \leq c_{t} \leq d_{t}, y_{t} \leq h_{t}$, and $0 \leq q_{t} \leq n_{t}$ for those agents who are matched in the first subperiod of period $t$ and $q_{t}=n_{t}=0$ for those agents who are not. Under these constraints, the planner's problem consists of finding a feasible plan $\left\{c_{t}, q_{t}\right\}_{t=0}^{\infty}$ such that

$$
\begin{aligned}
& \liminf _{T \rightarrow \infty} E_{0} \sum_{t=0}^{T}\left\{\beta^{t}\left\{\alpha\left[u\left(q_{t}\right)-q_{t}\right]+U\left(c_{t}\right)\right\}\right. \\
&\left.-\beta^{t}\left\{\alpha\left[u\left(\tilde{q}_{t}\right)-\tilde{q}_{t}\right]+U\left(\tilde{c}_{t}\right)\right\}\right\} \geq 0
\end{aligned}
$$

for all feasible plans $\left\{\tilde{c}_{t}, \tilde{q}_{t}\right\}_{t=0}^{\infty}$. Here, $E_{0}$ denotes the expectation with respect to the probability measure over sequences of dividend realizations induced by $F$. The solution is $\left\{c_{t}, q_{t}\right\}_{t=0}^{\infty}=\left\{x_{t}, q^{*}\right\}_{t=0}^{\infty}$. In equilibrium, $c_{t}=x_{t}$-that is, the equilibrium consumption of fruit is at the efficient level. However, the equilibrium allocation has $q_{t} \leq q^{*}$, which may hold with strict inequality in some states. That is, in a monetary equilibrium, consumption and production in the decentralized market may be below their efficient levels.

It is convenient to introduce the following notion of a nominal interest rate before stating the results. Consider an illiquid nominal bonda one-period, risk-free government bond that pays a unit of money in the centralized market 
and cannot be used in decentralized exchange. Let $\phi_{t}^{n}$ denote the price of this asset. In equilibrium, this price must satisfy $U^{\prime}\left(x_{t}\right) \phi_{t}^{n}=$ $\beta E_{t}\left[U^{\prime}\left(x_{t+1}\right) \phi_{t+1}^{m}\right]$. Since $\phi_{t}^{n} / \phi_{t}^{m}$ is the money price of a nominal bond, the (net) nominal interest rate in a monetary equilibrium is $i_{t}=\phi_{t}^{m} / \phi_{t}^{n}-1$ or, equivalently,

$$
i_{t}=\frac{E_{t}\left[L\left(\Lambda_{t+1}\right) \lambda_{t+1}^{m}\right]}{E_{t}\left(\lambda_{t+1}^{m}\right)}-1
$$

Proposition 1 Equilibrium quantities in a monetary equilibrium are Pareto optimal if and only if $i_{t}=0$ with probability 1 , for all $t$.

Proposition 1 establishes the optimality of the Friedman rule-Milton Friedman's (1969) prescription that monetary policy should induce a zero nominal interest rate to lead to an optimal allocation of resources. The proof is as follows: The equilibrium allocation is efficient if and only if $q_{t}\left(\Lambda_{t}\right)=q^{*}$, and this equality holds if and only if $\Lambda_{t} \geq q^{*}$-that is, if and only if the real value of the equilibrium portfolio, $\Lambda_{t}$, is at least as large as the real liquidity needs, represented by $q^{*}$. The nominal interest rate, $i_{t}$, is zero if and only if $L\left(\Lambda_{t+1}\right)=1$, and this equality holds if and only if $\Lambda_{t} \geq q^{*}$. Hence, $q_{t}\left(\Lambda_{t}\right)=q^{*}$ if and only if $i_{t}=0$. Intuitively, the cost of producing real balances is zero to the government, so the optimum quantity of real balances should be such that the marginal benefit-which in equilibrium equals the marginal cost, $i_{t}$-is zero to the economic agents.

I next turn to the question of implementation: Which monetary policies are consistent with a monetary equilibrium in which the nominal interest rate is at its optimal target level of zero? The following result addresses the issue of (weak) implementation by characterizing a family of monetary policies that are consistent with an equilibrium with $i_{t}=0$ for all $t$.

\section{Proposition 2}

$$
\text { Let } \phi_{t}^{s *}=E_{t} \sum_{j=1}^{\infty} \beta^{j} \frac{U^{\prime}\left(x_{t+j}\right)}{U^{\prime}\left(x_{t}\right)} x_{t+j},
$$

$\lambda_{t}^{s^{*}}=U^{\prime}\left(x_{t}\right)\left(\phi_{t}^{s^{*}}+x_{t}\right)$, and $\mathcal{T}$ be the set of dates for which $q^{*}-\lambda_{t}^{s^{*}}>0$ holds with probability $\pi_{t}>0$.
Assume that inf $f_{t \in \mathcal{T}} \pi_{t}>0$. A monetary equilibrium with $i_{t}=0$ with probability 1 for all $t$ exists under a deterministic money supply process $\left\{M_{t}\right\}_{t=0}^{\infty}$ if and only if the following two conditions hold:

(3) $\lim _{t \rightarrow \infty} M_{t}=0$

(4) $\inf _{t \in \mathcal{T}} M_{t} \beta^{-t}>0$ if $\mathcal{T} \neq \varnothing$.

Conditions (3) and (4) are rather unrestrictive asymptotic conditions. Condition (3) requires that the money supply converges to zero. Condition (4) requires that asymptotically, on average over the set of dates $\mathcal{T}$ when fiat money plays an essential role, the growth rate of the money supply must be at least as large as the rate of time preference. Versions of this result have been proven by Wilson (1979) and Cole and Kocherlakota (1998) for deterministic competitive economies with cash-in-advance constraints that are imposed on agents every period with probability 1 .

Proposition 2 has several implications. First, even though liquidity needs are stochastic in this environment (because equity, whose value is stochastic, can be used alongside money as a means of payment), a deterministic money-supply sequence can suffice to implement a zero nominal rate in every state of the world. Second, even within the class of deterministic monetary policies, there is a large family of policies that can implement the Pareto optimal equilibrium. Finally, it would be impossible for someone with access to a finite time-series for the path of the money supply to determine whether an optimal monetary policy is being followed. On the other hand, a single observation of a positive nominal rate constitutes definitive evidence of a deviation from an optimal monetary policy.

\section{POSITIVE RESULTS: INTEREST RATE TARGETS AND ASSET PRICES}

In this section, I consider perturbations of the optimal monetary policy that consist of targeting a constant positive nominal interest rate, and then discuss some positive implications of changes in the nominal interest rate target for the inflation 
rate, equity prices, and equity returns. To this end, it is convenient to focus on a recursive formulation in which prices are invariant functions of the aggregate state $\boldsymbol{s}_{t}=\left(x_{t}, M_{t}\right)$-that is, $\phi_{t}^{s}=\phi^{s}\left(\boldsymbol{s}_{t}\right)$, $\phi_{t}^{m}=\phi^{m}\left(\boldsymbol{s}_{t}\right)$, and $\boldsymbol{\lambda}_{t}=\left(\lambda^{s}\left(\boldsymbol{s}_{t}\right), \lambda^{m}\left(\boldsymbol{s}_{t}\right)\right)$, where $\lambda^{s}\left(\boldsymbol{s}_{t}\right)=$ $U^{\prime}\left(x_{t}\right)\left[\phi^{s}\left(\boldsymbol{s}_{t}\right)+x_{t}\right], \lambda^{m}\left(\boldsymbol{s}_{t}\right)=U^{\prime}\left(x_{t}\right) \phi^{m}\left(\boldsymbol{s}_{t}\right)$, and $\Lambda_{t}=$ $\lambda^{s}\left(\boldsymbol{s}_{t}\right)+\lambda^{m}\left(\boldsymbol{s}_{t}\right) M_{t}$. Also, I restrict attention to stationary monetary policies-that is, $\mu: \Xi \rightarrow \mathbb{R}^{+}$, so that $M_{t+1}=\mu\left(x_{t}\right) M_{t}$. To illustrate the main ideas as simply as possible, the following proposition focuses the analysis on the case of i.i.d. dividends and liquidity constraints that would bind with probability 1 at every date in the absence of money.

Proposition 3 Assume $d F\left(x^{\prime}, x\right)=d F(x)$. Let $l(\delta)=$ $1-\alpha+\alpha u^{\prime}\left(\delta q^{*}\right)$ and $\underline{\delta}$ be defined by $l(\underline{\delta})=1 / \beta$. Let $\delta_{0} \in(\underline{\delta}, 1)$ be given, and suppose that $B \leq$ $\left[1-\beta l\left(\delta_{0}\right)\right] \delta_{0} q^{*}$. Then for any $\delta \in\left[\delta_{0}, 1\right]$, there exists a recursive monetary equilibrium under the monetary policy

$$
\begin{aligned}
& \mu(x ; \delta)= \\
& \beta I(\delta) \frac{\delta q^{*}-\frac{1}{1-\beta l(\delta)} \int x^{\prime} U^{\prime}\left(x^{\prime}\right) d F\left(x^{\prime}\right)}{\delta q^{*}-\frac{\beta l(\delta)}{1-\beta l(\delta)} \int x^{\prime} U^{\prime}\left(x^{\prime}\right) d F\left(x^{\prime}\right)-x U^{\prime}(x)} .
\end{aligned}
$$

The equilibrium prices of equity and money are

$$
\phi^{s}(x ; \delta)=\frac{\beta l(\delta)}{1-\beta l(\delta)} \frac{\int x^{\prime} U^{\prime}\left(x^{\prime}\right) d F\left(x^{\prime}\right)}{U^{\prime}(x)} \text { and }
$$

$$
\phi^{m}(\boldsymbol{s} ; \delta)=
$$

$$
\frac{\delta q^{*}-\frac{\beta l(\delta)}{1-\beta l(\delta)} \int x^{\prime} U^{\prime}\left(x^{\prime}\right) d F\left(x^{\prime}\right)-x U^{\prime}(x)}{U^{\prime}(x) M} .
$$

Together with equation (2), the asset prices in equations (6) and (7) imply that the monetary policy (equation (5)) induces an equilibrium gross nominal interest rate that is constant (independent of $\boldsymbol{s}$ ) and equal to $I(\delta) \geq 1$ (with equality only if $\delta=1)$. The function $\mu(\cdot ; \delta)$ defines a class of monetary policies indexed by the parameter $\delta$, which effectively determines the level of the constant nominal interest rate implemented by the policy. According to equation (7), real money balances and the value of money are decreasing in the nominal interest rate target (increasing in $\delta$ ).
According to equation (6), under the proposed policy, the real price of equity is increasing in the nominal interest rate target (decreasing in $\delta$ ). As $\delta \rightarrow 1, l(\delta) \rightarrow 1$, and therefore (according to Proposition 1$)$ the policy $\mu(x ; \delta)$ approaches an optimal policy under which the recursive monetary equilibrium decentralizes the Pareto optimal allocation.

Notice that $\phi^{s}(x ; 1)$ is the "fundamental" equilibrium equity price that would result in a Lucas (1978) economy with no liquidity needs. Therefore, the fact that $\phi^{S}(x ; 1)<\phi^{S}(x ; \delta)$ for all $x$ and any $\delta \in\left[\delta_{0}, 1\right)$ implies that deviations from the optimal policy "inflate" real asset prices above the value that a financial analyst would calculate based on the expected stream of dividends discounted by the Lucas stochastic discount factor, $\beta U^{\prime}\left(x_{t+1}\right) / U\left(x_{t}\right)$.

On average, liquidity considerations generate a negative relationship between the nominal interest rate (and the inflation rate) and equity returns: If the target nominal rate, $I(\delta)-1$, is higher, the average inflation rate is higher, real money balances are lower, and the liquidity return on equity rises, which causes its price to rise and its measured real rate of return to fall. Intuitively, a higher nominal interest rate target implies that buyers are on average short of liquidity, so equity becomes more valuable as it is used by buyers to relax their trading constraints. This additional liquidity value causes the real financial return on equity to be lower, on average, at a higher interest rate.

Proposition 3 also shows explicitly how monetary policy must be conducted to support a recursive monetary equilibrium with a constant nominal interest rate (with the Pareto optimal equilibrium in which the nominal rate is zero as a special case): The growth rate of the money supply must be relatively low following states in which the real value of the equilibrium equity holdings is below average. Equivalently, the implied inflation rate will be relatively low between state $x$ and a next-period state $x^{\prime}$, if the realized real value of the equilibrium equity holdings in state $x$ is below the state- $x$ conditional expectation of its value next period. 


\section{CONCLUSION}

I have presented a simple version of a prototypical search-based monetary model in which money coexists with a financial asset that yields a risky real return. In this formulation, money is not assumed to be the only asset that must, nor the only asset that can, play the role of a medium of exchange: Nothing in the environment prevents agents from using equity along with money, or instead of money, as a means of payment. Since the equity share is a claim to a risky aggregate endowment, the fact that agents can use equity to finance purchases implies that they face aggregate liquidity risk, in the sense that in some states of the world, the value of equity holdings may ultimately be too low relative to what would be needed to carry out the transactions that require a medium of exchange. This seems like a natural starting point to study the role of money and monetary policy in providing liquidity to lubricate the mechanism of exchange in modern economies.
In this context, I characterized a large family of optimal monetary policies. Every policy in this family implements Friedman's prescription of zero nominal interest rates. Under an optimal policy, equity prices and returns are independent of monetary considerations. I have also studied a class of monetary policies that target a constant, but nonzero, nominal interest rate. For this perturbation of the family of optimal policies, I found that the model articulates the idea that, to the extent that a financial asset is valued as a means to facilitate transactions, the asset's real rate of return will include a liquidity return that depends on monetary considerations. As a result of this liquidity channel, persistent deviations from the optimal monetary policy will cause the real prices of assets that can be used to relax borrowing or other trading constraints to exhibit persistent deviations from their fundamental values.

\section{REFERENCES}

Cole, Harold L. and Kocherlakota, Narayana. “Zero Nominal Interest Rates: Why They're Good and How to Get Them.” Federal Reserve Bank of Minneapolis Quarterly Review, Spring 1998, 22(2), pp. 2-10.

Friedman, Milton. “The Optimum Quantity of Money,” in The Optimum Quantity of Money and Other Essays. Chap. 1. Chicago: Aldine, 1969, pp. 1-50.

Lagos, Ricardo. “Asset Prices and Liquidity in an Exchange Economy.” Staff Report 373, Federal Reserve Bank of Minneapolis, May 2006; www.minneapolisfed.org/research/SR/SR373.pdf.

Lagos, Ricardo. “Asset Prices, Liquidity, and Monetary Policy in an Exchange Economy.” Working paper, New York University, April 2009.

Lagos, Ricardo. "Some Results on the Optimality of the Friedman Rule in the Search Theory of Money." Journal of Economic Theory, 2010 (forthcoming).

Lagos, Ricardo and Wright, Randall. “A Unified Framework for Monetary Theory and Policy Analysis.” Journal of Political Economy, June 2005, 113(3), pp. 463-84.

Lucas, Robert E. Jr. “Asset Prices in an Exchange Economy.” Econometrica, November 1978, 46(6), pp. 1426-45.

Wilson, Charles. “An Infinite Horizon Model with Money,” in Jerry R. Green and José A. Scheinkman, eds., General Equilibrium, Growth, and Trade. New York: Academic Press, 1979, pp. 79-104. 
